\title{
Endocardial lead extraction with transoesophageal echocardiography guidance for cardiovascular implantable electronic device (CIED) related infections: four cases report
}

Qiaoyu Han

Peking University People's Hospital

Yi Feng

Peking University People's Hospital

Hui Ju

Peking University People's Hospital

Yan Jiang

Peking University People's Hospital

Feng Ze

Peking University People's Hospital

Xuebin Li

Peking University People's Hospital

Luyang Jiang ( $\sim$ jiangluyang1018@sina.com )

Peking University People's Hospital https://orcid.org/0000-0002-4341-4444

\section{Case report}

Keywords: Lead extraction, TEE, Complication, TTE, CIED infection

Posted Date: February 24th, 2021

DOI: https://doi.org/10.21203/rs.3.rs-230838/v1

License: (c) (i) This work is licensed under a Creative Commons Attribution 4.0 International License. Read Full License 


\section{Abstract \\ Background}

With the expanding use of cardiac implantable electronic device (CIEDs) in older populations with more complicated conditions have bring about higher rates of CIED infections. The recommended treatment of which involves the complete removal of all hardware, followed by antibiotic therapy and re-implant. Application of transoesophageal echocardiography (TEE) helps improving efficacy and safety by capacitating the operators to perform the procedure, guiding them to better plans and to rapidly recognize and manage relative complications.

\section{Case presentation}

: We report four cases of CIED infections from single-centre, who were extracted endocardial leads with intra-Op TEE monitoring and guidance. Three of them had three fatal complications as pulmonary embolism, pericardial tamponade and tricuspid trauma. The other one was found a massive vegetation detected by TEE not by pre-op TTE, which avoided intra-op embolism.

\section{Conclusions}

Continuously intra-op TEE should be one of the routine monitoring methods in these high-risk endocardial leads extraction procedures.

\section{Background}

Over the past several decades, cardiovascular implantable electronic devices (CIEDs), including implantable pacemakers (PMs), implantable cardioverter-defibrillators (ICDs), and cardiac resynchronization therapy (CRT) devices, have become more and more important in cardiac disease management and have significantly improved patients' quality of life. $[1,2]$ The increased rate of CIED implantation as well as increased implantation in older populations with more complicated conditions have bring about higher rates of CIED infections. [3]

According to the reports, the overall incidence of CIED infections ranges between $0.5 \%$ and $4.0 \%$. [4-6] CIED infections related complications can be life-threatening, often requiring device removal. The recommended treatment of CIED infections, no matter systemic or localized to pocket, regardless of location (subcutaneous, transvenous, or epicardial), involves the complete removal of all hardware (generator and leads), followed by antibiotic therapy and re-implant. [7] If retain the hardware, the risk of infection recurrence is high. $[4,8,9]$

With the development of technology, there are several ways to extract leads. Transvenous lead extraction is feasible and widely used nowadays. Surgical extraction via thoracotomy or sternotomy with 
cardiopulmonary bypass (CPB) is supplemental method.

Most leads can be safely and completely removed transvenously. However, the leads may become endothelialized over time, which rendering removal process extremely difficult. Long-time implanted transvenous lead extraction remains a complex procedure with high risk and can be associated with major complications, including cardiac avulsion or tear, cardiac tamponade, vascular injury, pulmonary embolism, valve disruption or even cardiac arrest. [10-13]

Many of these major complications may be treatable or inevitable if identified immediately. Therefore, improved monitoring during this procedure is essential. Transoesophageal echocardiography (TEE) is a safe and highly sensitive technique in the intracardiac monitoring, which can provide a continuous and real-time monitoring of intracardiac structures as well as heart and valves functions during the extraction procedure. Application of TEE helps improving efficacy and safety by capacitating the operators to perform the procedure, guiding them to better plans and to rapidly recognize and manage relative complications. [12]

We present 4 case reports, in which TEE were used to guide the endocardial lead extraction procedure, hoping to highlight the value of TEE for early diagnosis and management of major complications associated with transvenous lead extraction in patients with CIED infection.

\section{Cases Presentation}

\section{Case 1}

was a 65-year-old male admitted for intermittent fever and chills, considering infective endocarditis (IE). The patient had undergone a permanent dual chamber (DDD) pacemaker implantation 7 years back for third degree atrioventricular block and complete left bundle branch block. Transthoracic echocardiogram (TTE) and TEE revealed multiple loose vegetation attached to the aortic valve $(4 * 6 \mathrm{~mm})$ and right atrium (RA) lead $(3.2 * 2.0 \mathrm{~cm}$ maximum), which traversed the tricuspid valve and entered the right ventricle (RV), swinging between RA and RV with the heart cycle. No tricuspid regurgitation (TR) was seen. The patient initially underwent a transvenous lead extraction under monitored local anaesthesia in catheter lab. After a lead was extracted off, the patient had sudden cardiac arrest with suspected emergent pulmonary embolism by exfoliated vegetation. The cardiopulmonary resuscitation (CPR) and venography had taken, and the operator tried to extend a sheath guidance wire to the far distal of right ventricle outflow tract (RVOT) with the guidance of X-Ray, trying to push the embolus to the branch of pulmonary artery. The resuscitation was successfully performed after the embolism was push to the right pulmonary artery. The patient was transferred to the operating room (OR) for pulmonary embolectomy under general anaesthesia (GA) and TEE guidance.

TEE revealed that the vegetation was embolized to the opening of right pulmonary artery and TR in large quantities (Fig. 1,2 and Video 1). Following, his ECG showed a ST-T change, heart rate increased, arterial 
blood pressure dropped to $75 / 45 \mathrm{mmHg}$, blood oxygen saturation dropped to a minimum of $65 \%$, and $\mathrm{P}_{\mathrm{ET}} \mathrm{CO}_{2}$ decreased, considering pulmonary artery embolism.

Emergent pulmonary embolectomy and tricuspid valvuloplasty was undertaken with CPB and deep hypothermic circulatory arrest (DHCA). Incised the right pulmonary artery along its longitudinal axis, vegetation was seen embolized the origin of middle and lower pulmonary artery, the diameter of which was closed to $2.0 \mathrm{~cm}$ (Figure 3 ). Completely removed the vegetation and small emboli in the distant artery branches. Inspecting the tricuspid valve ring was expanded and anterior leaflet was prolapsed, so a tricuspid annuloplasty was undertaken. The rewarming and weaning from CPB process was smooth. Followed by sewing leads of epicardial temporary pacemaker, the pacing rhythm was $80 \mathrm{bpm}$.

After the surgery, the patient was transferred to cardiosurgery intensive care unit (CSICU) for continuous monitoring. He recovered well after the operation, extubated on the 6th postoperative day, off all vasopressors (dopamine and epinephrine) on the 9th day, ambulated on the 17th day. Postoperative echocardiography found no obvious abnormalities. A new permanent pacemaker (PPM) was implanted via the right subclavian vein 21 days later. The patient had shown no evidence of infection or complications during several months of follow up.

\section{Case 2}

was a 60-year-old male with a PPM for Brugada syndrome for 9 years, admitted for a 3-month history of pain and erythema over the site of the generator pocket. There was no evidence of systemic infection. A TTE was suggestive of slightly aortic valvular regurgitation (AR) and decreased diastolic function of left ventricular.

The patient underwent a transvenous lead extraction and generator pocket debridement under GA with TEE monitoring. With the help of Evolution Sheath, RA and RV leads were extracted successfully. After the leads extraction, the blood pressure of the patient suddenly decreased to $50 / 30 \mathrm{mmHg}$. Later on, his blood pressure could not be measured. By this time, TEE showed massive pericardial effusion, suggesting pericardial tamponade (Video 2).

Pericardiocentesis was done immediately and drained off about $150 \mathrm{ml}$ blood from the catheter. Dopamine and adrenaline were pushed intravenously. However, there were no any improvement. CPR was done at once (Video 3), while emergent exploratory thoracotomy was undertaken. After opening the chest, blue and purple pericardium was seen with high tension. When opening the pericardium, large amount of dark red blood sprayed out and much blood clots accumulated in the pericardial cavity. After removed all the blood clots and decompressed the heart, the patient's blood pressure back to $110 / 75 \mathrm{mmHg}$, the CPR was successful. Confirming that there was no active haemorrhage, surgeons placed the drainage tube and closed the chest.

Rechecked TEE, no progressive pericardial effusion was found (Video 4). The patient was transferred to CSICU. He was extubated 1 day later and recovered well. 


\section{Case 3}

was a 68-year-old female who had undergone a DDD pacemaker implantation 14 years ago for sick sinus syndrome (SSS), admitted for fever and chills for 1 month. A TTE was suggestive of slightly TR.

The patient underwent a transvenous lead extraction and generator pocket debridement under GA with TEE monitoring. After induction of GA, TEE showed slightly TR, the same as outcomes of preoperative TTE (Video 5). Atrial and ventricular leads were extracted successfully with the help of specialized locking style. After removal of all leads, TEE found TR dramatic increased with significant hepatic venous reversal wave (Figs. 4 and 5). The patient's hemodynamic status was unstable as well as the central venous pressure (CVP). The extracted lead had a tissue attached on consisted the tricuspid leaflet iatrogenic trauma (Video 6).

The patient was sent to OR for emergency tricuspid exploring under CPB. During CPB, surgeons found the tricuspid anterior leaflet were missing, where the severe TR was original from. Due to the anatomic structure of tricuspid were incomplete, a mechanical valve was replaced. The patient did not extubated and died of heart failure a week after surgery.

\section{Case 4}

was a 71-year-old female admitted for skin erosion at the site of the generator pocket for 7 days. The patient had undergone a DDD pacemaker implantation 22 years back for SSS.

She was reimplanted the generator and one of the leads 15 years ago, and was reimplanted the generator for the second time 6 years ago. A TTE was suggestive of ventricular septal thickening, mild AR, left ventricular diastolic function decrease, and pacing leads echo in the RA and RV without any vegetation was noticed (Video 7). The patient underwent a transvenous lead extraction and generator pocket debridement under GA. Intraoperative TEE revealed a large $(2.4 * 2.2 \mathrm{~cm})$ vegetation within the RA (Fig. 6 and Video 8), which was not match with the findings of pre-op TTE. Considering of the high risk of pulmonary embolism and other critical complications, the operators decided to suspend the surgery and planned to perform selective endocardial lead extraction and epicardium temporary pacing leads implantation.

During the selective operation, surgeons found that the RA lead was curly with a massive vegetation attached (Fig. 7), which was consistent with the findings of TEE. Besides, RV leads were also surrounded by vegetation. After separated and complete extracted the leads, an epicardium temporary pacemaker was implanted. The whole procedure was difficult, but smooth. No major complications occurred. The patient recovered well after the operation.

\section{Discussion}

CIEDs infections including pocket infections, valvular endocarditis, lead endocarditis, or sepsis. [10] All these four cases are endocardial lead extraction for CIED infectious patients suffering from IE which 
original from local pocket infection. Most of those patients are in their old age suffering from long-term fever with variety of complications: heart failure, chronic anaemia, hypo leukopenia, coagulation dysfunction as well as in very poor health conditions.

Transvenous lead extraction is the most commonly used method now. It is reported that with current techniques, complete lead extraction can be achieved in more than $90 \%$ of cases, with a major complication rate of less than $2 \%$ and mortality rate of less than 1\%. [14] However, a major factor associated with increased risk of complications is the time from implantation. The longer the leads have been implanted, the more likely those patients are to either have a failed lead extraction or complications. [15] This may due to increased development of fibrotic tissue around the body of the leads over time after the lead implantation. With excessive fibrotic or scar tissue, the leads may have more difficulty being separated and thus can cause vascular or myocardial injury. [16] In our four cases, the time of patients from lead implantation was $7,9,14$ and 22 years, which may increase the difficulty of lead extraction, and may have high risks of complications. Considering of the difficulty and high risks of the procedure, in these four cases, lead extractions were performed under GA with TEE guidance.

Complications occurred in first three cases. Major complications occurred in first two cases that need another emergent open-heart operation. After lead extraction and device removal, the patient of case 1 had symptoms compatible with pulmonary embolism, the patient of case 2 had clinical manifestations of cardiac tamponade, tricuspid valve injury was seen in case 1 and case 3 . In the last case, there was a new discovering of a large vegetation within RA during transvenous lead extraction, then performed a selective operation without any complication occurred. In these cases, four significant findings discerned by TEE during transvenous lead extraction: pulmonary embolism, pericardial effusion or cardiac laceration, TR, and intracardiac mass.

As a convenient and efficient monitoring tool, TEE can monitor and offer helpful informations, including but not limited to wall motion, valvular function, and possible lead vegetations throughout the procedure. This may help early detection and diagnosis of complications. In case 1 and 2 , the embolus or pericardial effusion was observed visually through TEE, which helped to identify the diagnosis and dispose of complications in time. Rapid detection and treatment are critical for improving chances of survival. Thus, these two patients have good prognosis.

Lead extraction with the guidance of TEE is also reported in some other studies. Endo et al [12] reviewed the records of 108 patients who underwent transvenous lead extraction with TEE guidance to assess the utility of TEE during the procedure. They found that useful clinically findings were provided by TEE in $16 \%$ of cases, which led to a converted operation or an open surgery. Believed that TEE during transvenous lead extraction could provide valuable real-time information that improved efficacy and safety.

Tricuspid valve injury during lead extraction procedures with incidence rates of $3.5-12 \%$. $[12,17,18]$ According to statistics, TR seems to be a rare complication but can lead to severe clinical consequences such as right heart failure. [19] Tricuspid valve injury and a new or worsened TR were seen in both case 1 and 3 after removal of all hardware. However, TEE revealed that the level of TR was more serious in 
patient of case 1 than case 3 . In case 1, the new occurred TR affected the patient's haemodynamics, while the patient of case 3 still had stable vital signs in spite of worsened TR. So treatments to these two patients were different: Patient of case 1 was undertaken an emergent tricuspid valvuloplasty, while in case 3, operators did not deal with it and decided to observe for more time. TEE may benefit the patients by assisting operators to make an optimal decision.

Besides, the sensitivity of TEE to vegetations is much higher than TTE. In case 4, intraoperative TEE revealed a large vegetation within the RA, while the preoperative TTE showed leads without any vegetation. If TEE was not performed in this case, the risk of pulmonary embolism during the procedure would be high. Before, Rodriguez Y et al [20] compared the TTE and TEE results of 150 patients. From these 150 patients, 55 had vegetations detected by TEE-these findings were corroborated by intraoperative observation and post-operative pathology. Only 14 of these 55 patients had vegetations detected by TTE. Therefore, the sensitivity of the TTE was calculated at $25.5 \%$. That means the missed diagnosis rate was as high as $74.5 \%$. Vegetations are visualized in nearly $90 \%$ of patients with definite IE. In our experience, the cut off of transvenous extraction of vegetation is $\leq 20 \mathrm{~mm}$ since the first case pulmonary embolism was happed. Massive vegetation (Diameter $>20 \mathrm{~mm}$ ) has to remove by surgery because of the risk of embolization and hemodynamic corruption [21]. In the last case, the pre-Op TTE did not inform any massive vegetation. But the intra-Op TEE showed a massive vegetation which consisted with surgical exploring result. If the TEE did not be performed, severe complications as embolism might occurred.

To sum up, TEE has the following advantages in endocardial lead extraction: (1) TEE is a safe tool that can provide continuous and real-time intraoperative monitoring. Giving essential informations of major complications, helping early diagnosis and in time management, so as to improve patent's prognosis; (2) TEE can help the operators making optimal next plans and provide a guidance or reference for followed treatments; (3) TEE is more sensitive than TTE. It can provide clearer and higher sensitive images for the heart structures, valves functions and endocardial vegetations. Therefore, the use of TEE in lead extraction is practical and meaningful.

We present four case reports underling the value of TEE for early recognition and management of major complications associated with lead extraction. The sample size was limited and originated from a single centre. A large population from multi-centre for a randomized controlled study could be conducted to increase the generalizability of the findings.

\section{Conclusion}

With increasing utilization of CIEDs and rising rate of CIEDs infections, it can be expected that the number of leads extraction will sustainable growth. As patients with CIEDs live longer, implanted leads may become more difficult to explant. Anaesthesiologists should make full use of TEE, as which is a safe and highly sensitive advanced haemodynamic monitoring and imaging technique, in order to ensure the best outcomes of the patients. 


\section{Abbreviations}

CIEDs: cardiovascular implantable electronic devices; PPM: permanent pacemaker; ICDs: implantable cardioverter-defibrillators; CRT: cardiac resynchronization therapy; CPB: cardiopulmonary bypass; TEE: transesophageal echocardiography; IE: infective endocarditis; DDD: dual chamber pacemaker; TTE: transthoracic echocardiogram; RA: right atrium; RV: right ventricle; TR: tricuspid regurgitation; CPR: cardiopulmonary resuscitation; RVOT: right ventricle outflow tract; OR: operating room; GA: general anaesthesia; DHCA: deep hypothermic circulatory arrest; CSICU: cardiosurgery intensive care unit; AR: aortic valvular regurgitation; SSS: sick sinus syndrome;

CVP: central venous pressure.

\section{Declarations}

\section{Ethics approval and consent to participate}

Not applicable.

\section{Consent for publication}

Informed consent for publication of completely anonymous data was obtained from the patient.

\section{Availability of data and materials}

Original data files are available upon request.

\section{Competing interests}

The authors declare no conflict of interest.

\section{Funding}

No funds were received for the manuscript's preparation.

\section{Authors' contributions}

LJ conceived and designed the study. QH and LJ drafted the manuscript. HJ, YJ and LJ edited the ultrasound images and critical reviewed the manuscript. All authors contributed to manuscript revision, read and approved the final manuscript.

\section{Acknowledgements}

None.

\section{References}


1. Chaudhry UA, Harling L, Ashrafian H, Athanasiou C, Tsipas P, Kokotsakis J, Athanasiou T: Surgical management of infected cardiac implantable electronic devices. Int J Cardiol 2016, 203:714-721.

2. Lambert CT, Tarakji KG: Cardiac implantable electronic device infection. Cleve Clin J Med 2017, 84(12 Suppl 3):47-53.

3. Baddour LM, Epstein AE, Erickson CC, Knight BP, Levison ME, Lockhart PB, Masoudi FA, Okum EJ, Wilson WR, Beerman LB et al: Update on cardiovascular implantable electronic device infections and their management: a scientific statement from the American Heart Association. Circulation 2010, 121(3):458-477.

4. Nielsen JC, Gerdes JC, Varma N: Infected cardiac-implantable electronic devices: prevention, diagnosis, and treatment. Eur Heart J 2015, 36(37):2484-2490.

5. Sandoe JA, Barlow G, Chambers JB, Gammage M, Guleri A, Howard P, Olson E, Perry JD, Prendergast $\mathrm{BD}$, Spry MJ et al: Guidelines for the diagnosis, prevention and management of implantable cardiac electronic device infection. Report of a joint Working Party project on behalf of the British Society for Antimicrobial Chemotherapy (BSAC, host organization), British Heart Rhythm Society (BHRS), British Cardiovascular Society (BCS), British Heart Valve Society (BHVS) and British Society for Echocardiography (BSE). J Antimicrob Chemother 2015, 70(2):325-359.

6. Biffi M: The never-ending story of CIED infection prevention: Shall we WRAP-IT and go. J CardiovasC Electrophysio/ 2019, 30(8):1191-1196.

7. Aljabri K, Garlitski A, Weinstock J, Madias C: Management of Device Infections. Card Electrophysiol Clin 2018, 10(1):153-162.

8. Goya M, Nagashima M, Hiroshima K, Hayashi K, Makihara Y, Fukunaga M, An Y, Ohe M, Yamazato S, Sonoda $\mathrm{K}$ et al: Lead extractions in patients with cardiac implantable electronic device infections: Single center experience. J Arrhythm 2016, 32(4):308-312.

9. Sohail MR, Uslan DZ, Khan AH, Friedman PA, Hayes DL, Wilson WR, Steckelberg JM, Stoner S, Baddour LM: Management and outcome of permanent pacemaker and implantable cardioverterdefibrillator infections. J Am Coll Cardiol 2007, 49(18):1851-1859.

10. Wilkoff BL, Love CJ, Byrd CL, Bongiorni MG, Carrillo RG, Crossley GH, Epstein LM, Friedman RA, Kennergren $\mathrm{CE}$, Mitkowski $\mathrm{P}$ et al: Transvenous lead extraction: Heart Rhythm Society expert consensus on facilities, training, indications, and patient management: this document was endorsed by the American Heart Association (AHA). Heart Rhythm 2009, 6(7):1085-1104.

11. Kennergren C, Bjurman C, Wiklund R, Gäbel J: A single-centre experience of over one thousand lead extractions. Europace 2009, 11(5):612-617.

12. Endo Y, O'Mara JE, Weiner S, Han J, Goldberger MH, Gordon GM, Nanna M, Ferrick KJ, Gross JN: Clinical utility of intraprocedural transesophageal echocardiography during transvenous lead extraction. J Am Soc Echocardiogr 2008, 21(7):861-867.

13. Sonny A, Wakefield BJ, Sale S, Mick S, Wilkoff BL, Mehta AR: Transvenous Lead Extraction: A Clinical Commentary for Anesthesiologists. J Cardiothorac Vasc Anesth 2018, 32(3):1101-1111. 
14. Madhavan M, Swale MJ, Gard JJ, Hayes DL, Asirvatham SJ: Contemporary pacemaker and ICD lead management: techniques for lead extraction. Expert Rev Cardiovasc Ther 2012, 10(7):875-887.

15. Kennergren C, Bucknall CA, Butter C, Charles R, Fuhrer J, Grosfeld M, Tavernier R, Morgado TB, Mortensen P, Paul V et al: Laser-assisted lead extraction: the European experience. Europace 2007, 9(8):651-656.

16. Bhatia M, Safavi-Naeini P, Razavi M, Collard CD, Tolpin DA, Anton JM: Anesthetic Management of Laser Lead Extraction for Cardiovascular Implantable Electronic Devices. Semin Cardiothorac Vasc Anesth $2017: 1089253217728581$.

17. Glover BM, Watkins S, Mariani JA, Yap S, Asta J, Cusimano RJ, Ralph-Edwards AC, Cameron DA: Prevalence of tricuspid regurgitation and pericardial effusions following pacemaker and defibrillator lead extraction. Int J Cardiol 2010, 145(3):593-594.

18. Franceschi F, Thuny F, Giorgi R, Sanaa I, Peyrouse E, Assouan X, Prévôt S, Bastard E, Habib G, Deharo $\mathrm{JC}$ : Incidence, risk factors, and outcome of traumatic tricuspid regurgitation after percutaneous ventricular lead removal. J Am Coll Cardiol 2009, 53(23):2168-2174.

19. Pecha S, Castro L, Gosau N, Linder M, Vogler J, Willems S, Reichenspurner H, Hakmi S: Evaluation of tricuspid valve regurgitation following laser lead extractiont. Eur J Cardiothorac Surg 2017, 51(6):1108-1111.

20. Rodriguez Y, Garisto J, Carrillo RG: Management of cardiac device-related infections: a review of protocol-driven care. Int J Cardiol 2013, 166(1):55-60.

21. Perez Baztarrica G, Gariglio L, Salvaggio F, Reolon E, Blanco N, Mazzetti H, Villecco S, Botbol A, Porcile R: Transvenous extraction of pacemaker leads in infective endocarditis with vegetations >/=20 mm: our experience. Clin Cardiol 2012, 35(4):244-249.

\section{Figures}




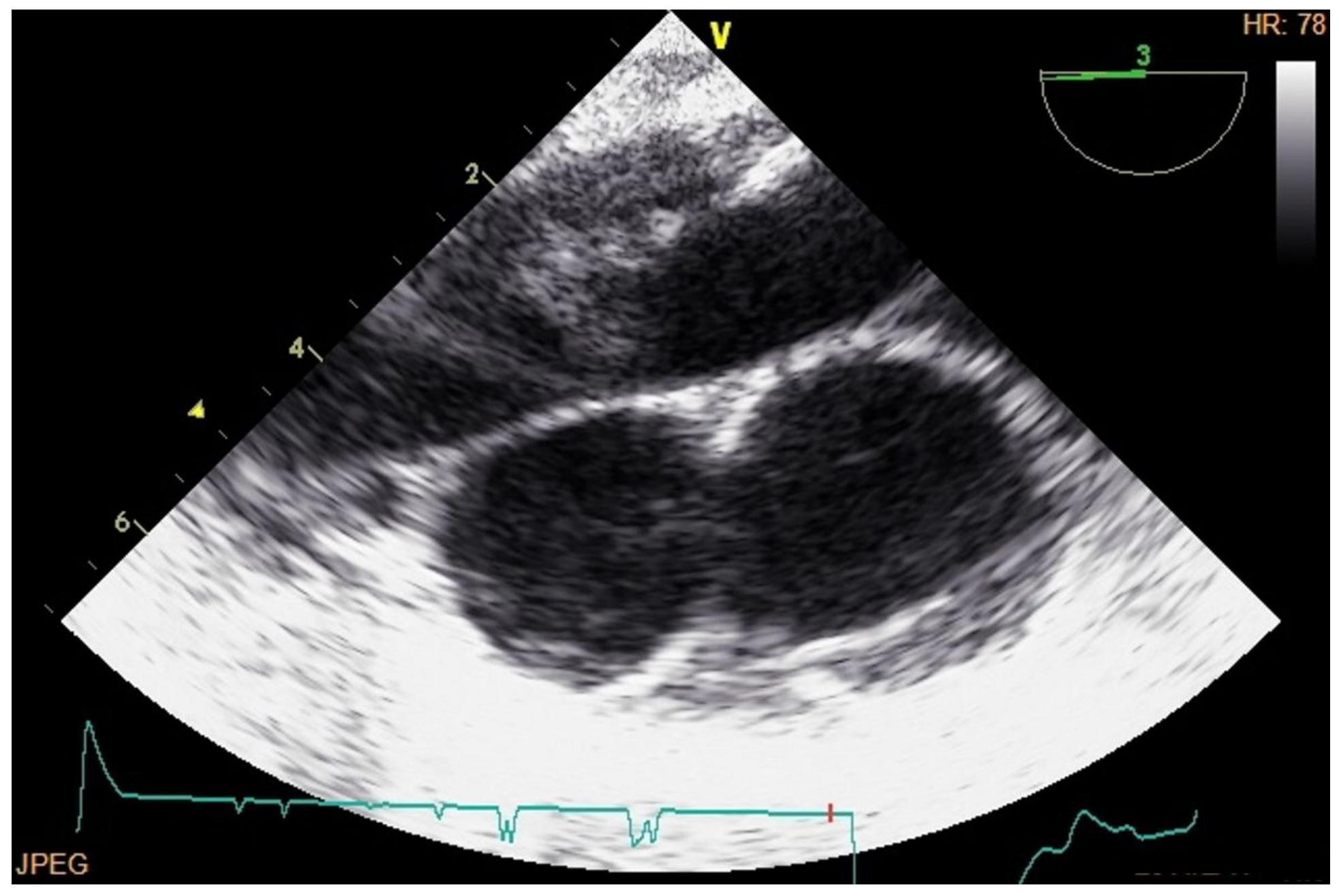

Figure 1

Case 1 Embolus and wire. 


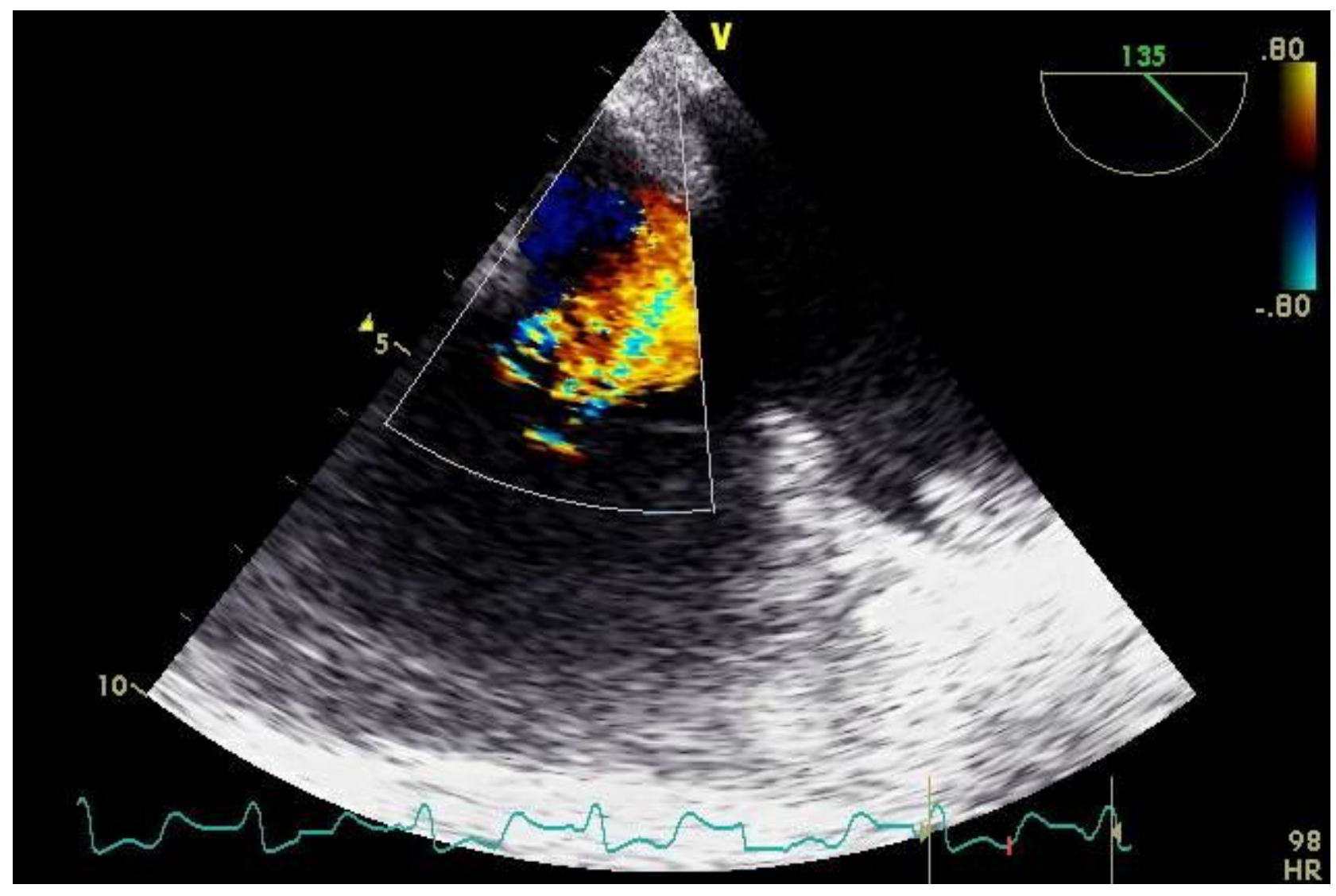

Figure 2

Case 1 Severe TR and Enlarged CS. 


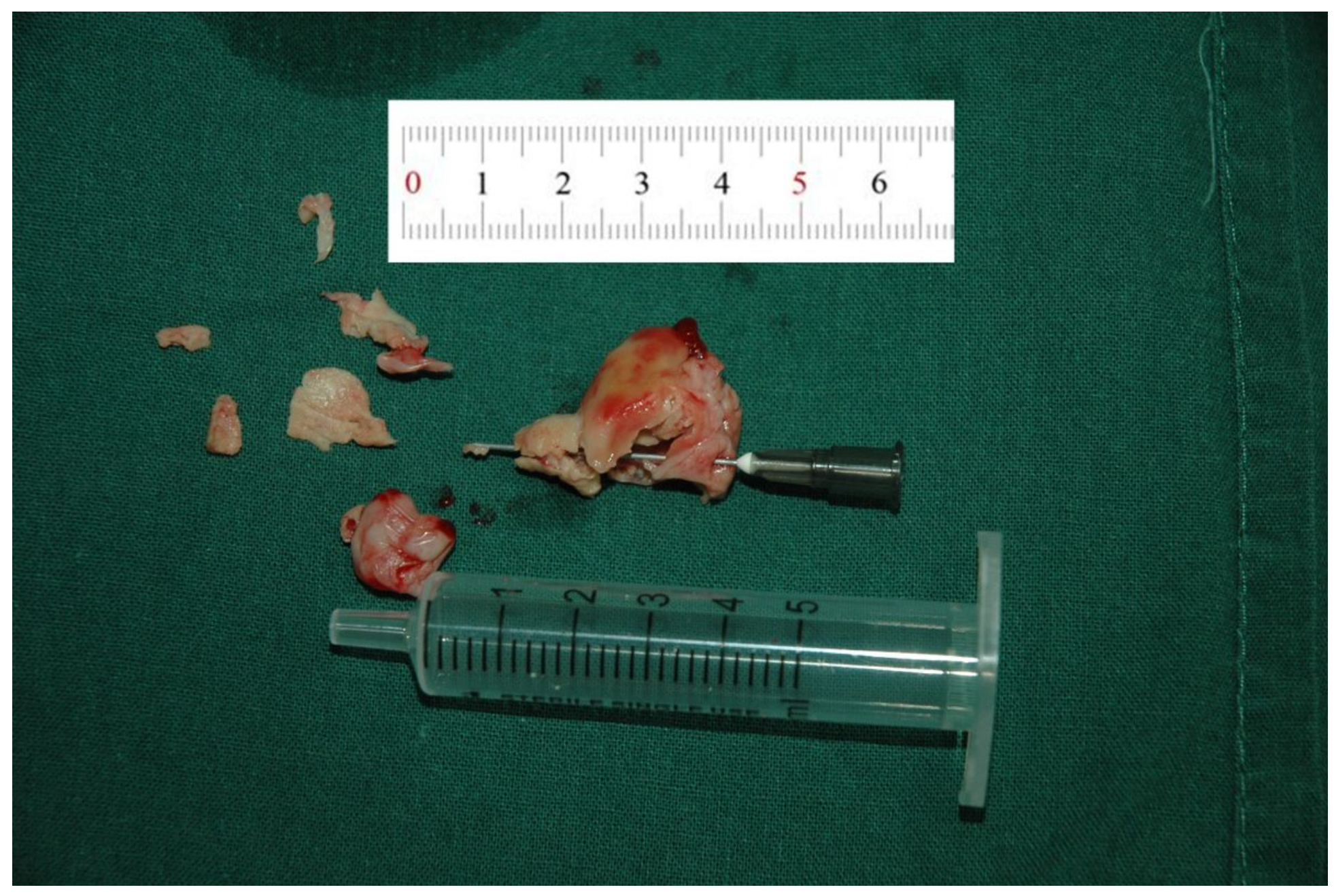

Figure 3

Case 1 Embolus. 


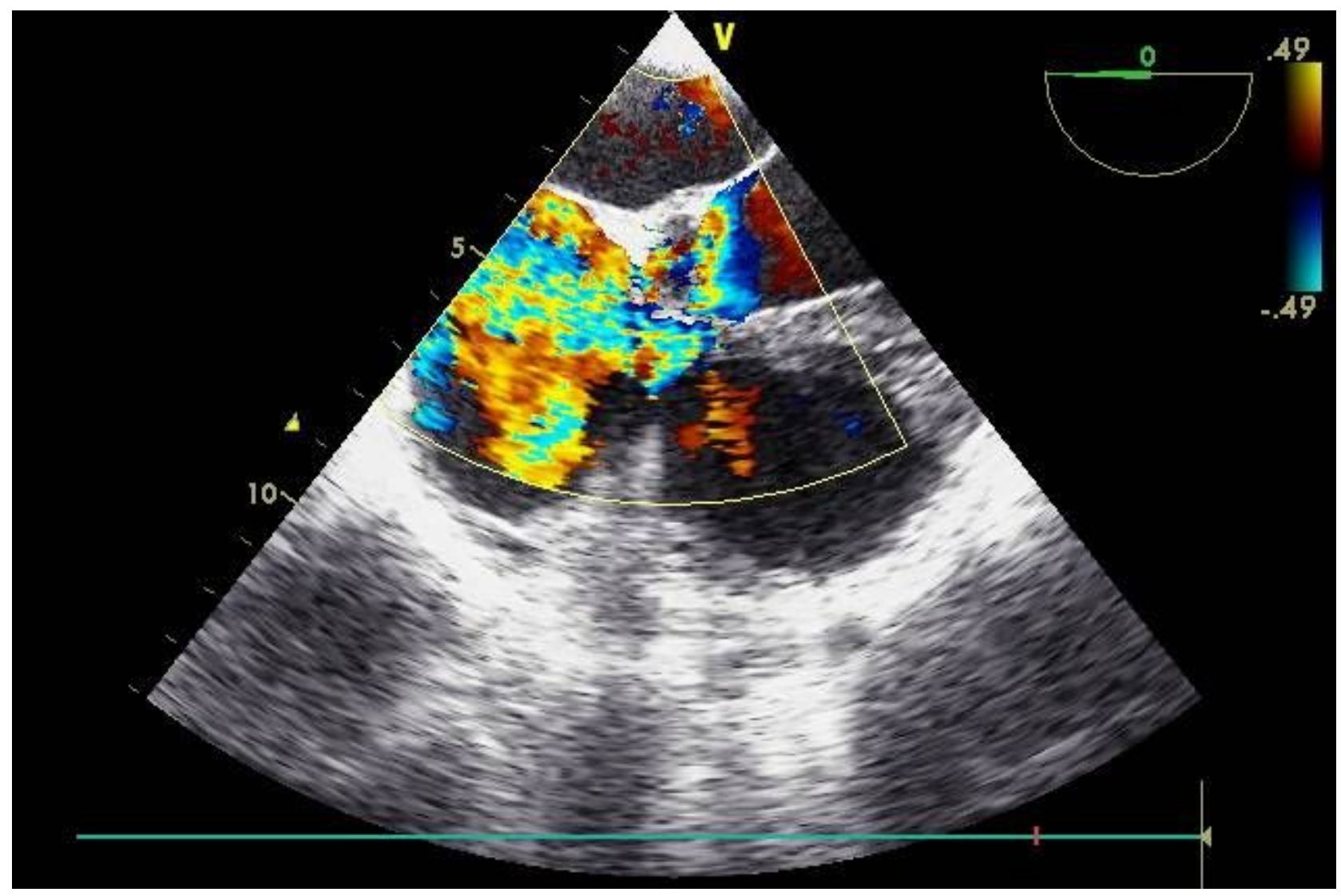

Figure 4

Case 3 Tricuspid regurgitation.

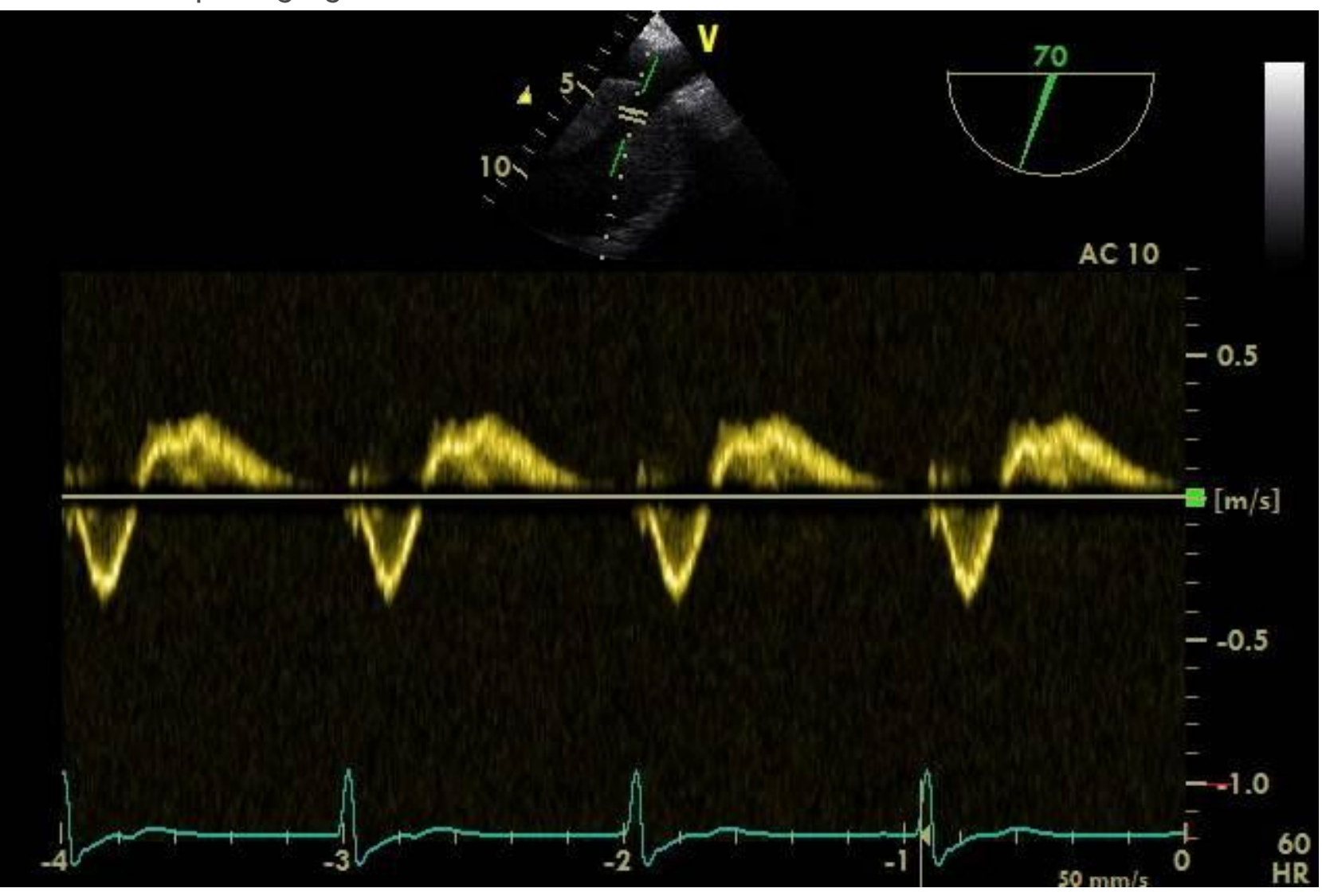


Figure 5

Case 3 Significant hepatic venous reversal wave.

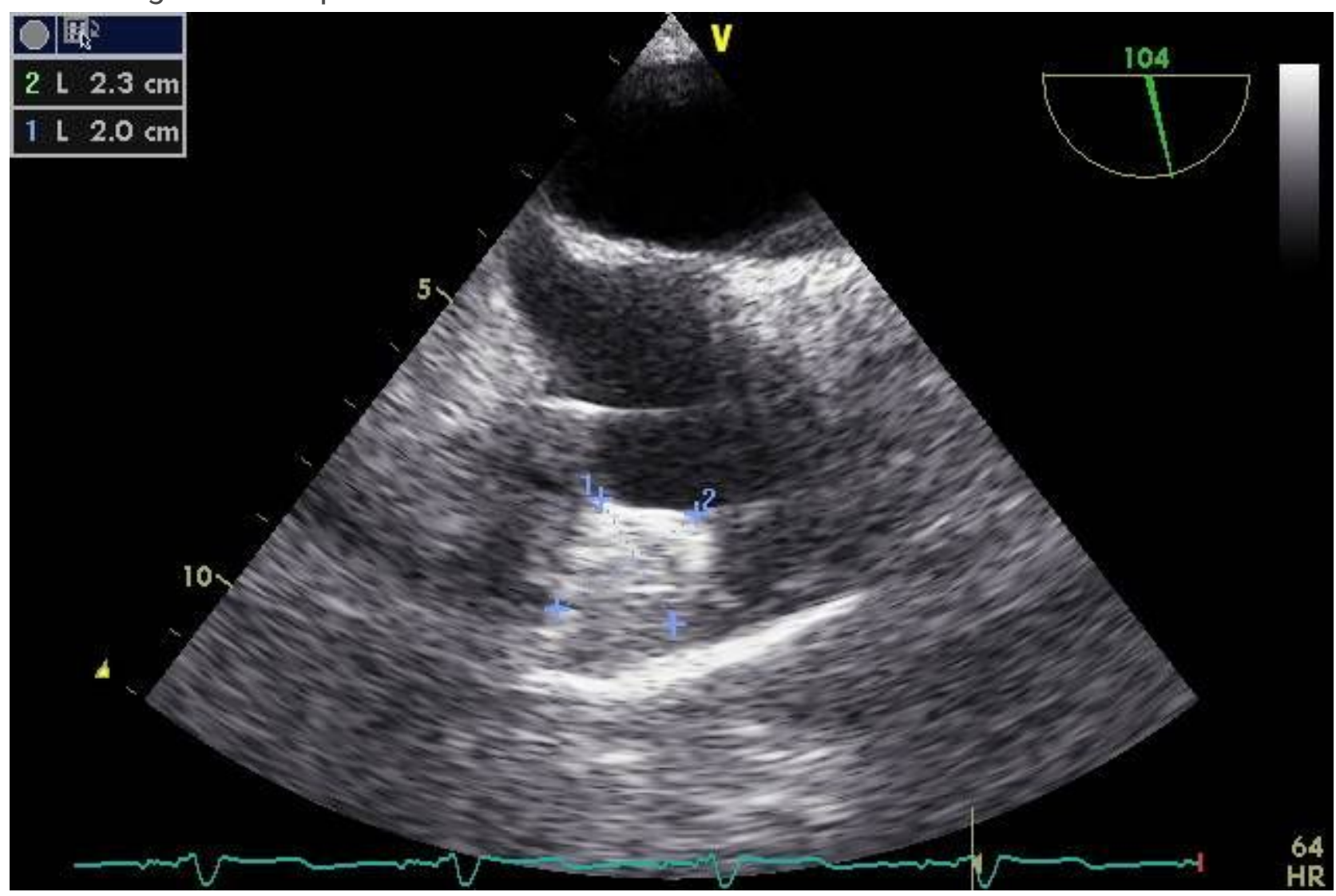

Figure 6

Case 4 Vegetation detected by TEE. 


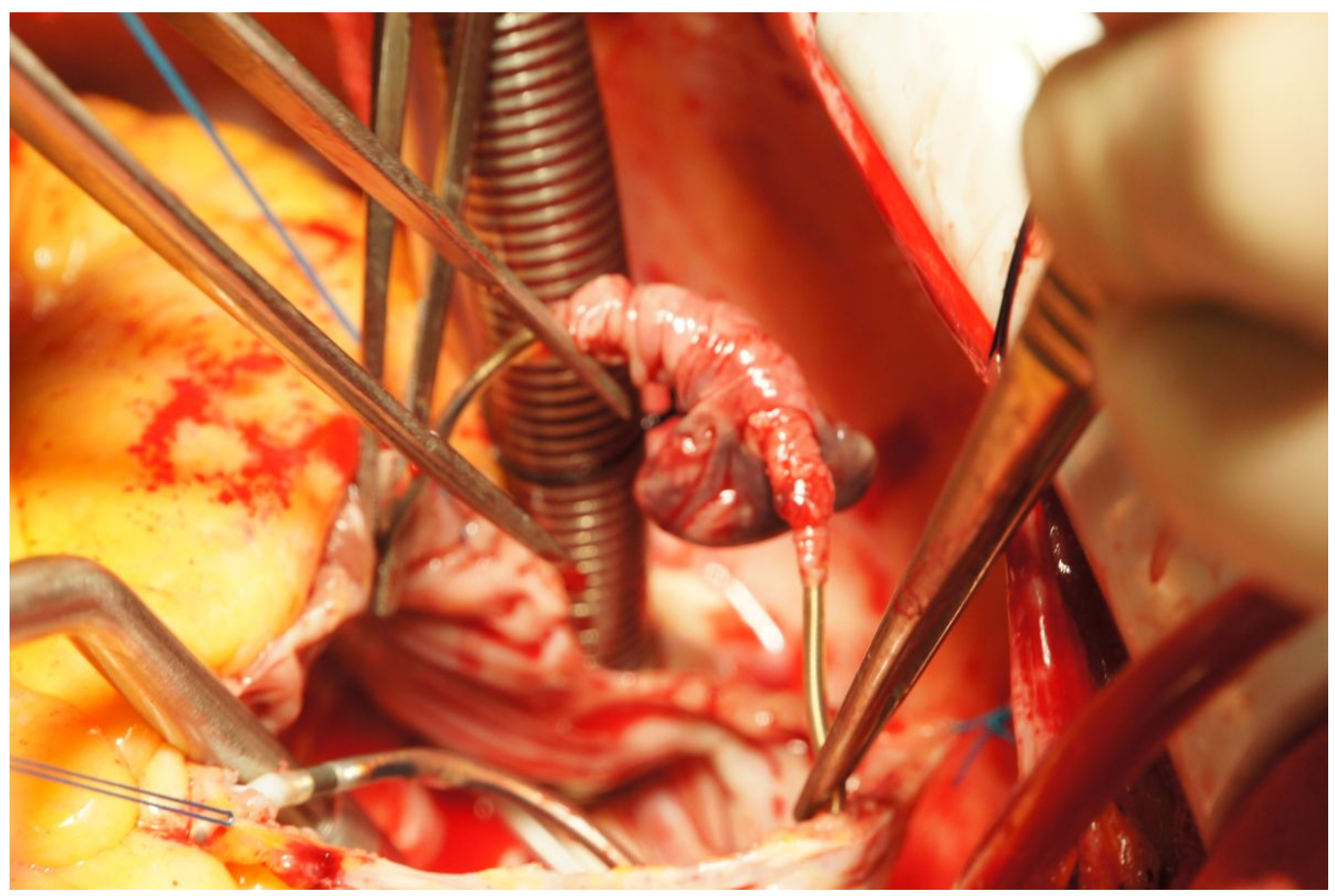

Figure 7

Case 4 a massive vegetation attached on the RA lead.

\section{Supplementary Files}

This is a list of supplementary files associated with this preprint. Click to download.

- Video1Case1Embolism.wmv

- Video2Case2Effusion.wmv

- Video3Case2Externalchestcompression.wmv

- Video4Case2Resuscitatedheart.wmv

- Video5Case3PreopslightlyTRwithleadwasseen.wmv

- Video6Case3Postoptricuspidleaflettraumaticprolapse.wmv

- Video7Case4PreTTEvegetationhadnotbeenshown.wmv

- Video8Case4VegetationwasshownbyTEE.wmv 